\title{
Paclitaxel encapsulated in cationic liposomes: A new option for neovascular targeting for the treatment of prostate cancer
}

\author{
CHRISTIAN BODE $^{1 *}$, LUTZ TROJAN $^{1 *}$, CHRISTEL WEISS $^{2}$, BETTINA KRAENZLIN $^{3}$, \\ UWE MICHAELIS $^{4}$, MICHAEL TEIFEL ${ }^{5}$, PETER ALKEN ${ }^{1}$ and MAURICE STEPHAN MICHEL ${ }^{1}$ \\ Departments of ${ }^{1}$ Urology, ${ }^{2}$ Medical Biometrics and Statistics, ${ }^{3}$ Medical Research Centre University Hospital Mannheim, \\ Theodor-Kutzer-Ufer 1-3, D-68161 Mannheim; ${ }^{4}$ MediGene AG, Lochhamer Str. 11, D-82152 Martinsried; \\ ${ }^{5}$ Æterna Zentaris GmbH, Weismuellerstr. 50, D-60314 Frankfurt am Main, Germany
}

Received January 9, 2009; Accepted February 13, 2009

DOI: $10.3892 /$ or_00000440

\begin{abstract}
Neovascular targeting is an established approach for the therapy of prostate cancer (PCa). Cationic liposomes have been shown to be absorbed by immature vascular endothelial cells due to negative electric charge of their outer cell membrane. We aimed to evaluate the antitumoural efficacy of paclitaxel encapsulated in cationic liposomes for the treatment of PCa. Tumours were generated by subcutaneous injection of $10^{6} \mathrm{MatLu}$ tumour cells into the right hind leg of 21 male Copenhagen rats. After tumour growth, the animals were treated by an i.v. infusion with either $5 \%$ glucose (Gl), paclitaxel (Pax), cationic liposomes (CL) or paclitaxel encapsulated in cationic liposomes (EndoTAG-1) on days 12, 14, 16 and 19. Treatment was initiated on day 12 after tumour inoculation at mean tumour volumes of $0.31 \pm 0.13 \mathrm{~mm}^{3}$. On the last day of treatment, animals treated with EndoTAG-1 had the significantly lowest tumour volumes with $2.49 \pm 0.84 \mathrm{~cm}^{3}$ vs. Pax $\left(5.59 \pm 0.45 \mathrm{~cm}^{3}\right)$ vs. CL $\left(3.87 \pm 1.25 \mathrm{~cm}^{3}\right)$ vs. GL $\left(5.17 \pm 1.70 \mathrm{~cm}^{3}\right)$. The quantification of MVD showed the lowest count for EndoTAG-1treated tumours $\left(11.78 \pm 2.68\right.$ vessels $\left./ \mathrm{mm}^{2}\right)$ followed by Gl $\left(15.64 \pm 6.68\right.$ vessels $\left./ \mathrm{mm}^{3}\right), \operatorname{Pax}\left(18.22 \pm 9.50\right.$ vessels $\left./ \mathrm{mm}^{3}\right)$ and CL $\left(40.9 \pm 32.8\right.$ vessels $\left./ \mathrm{mm}^{3}\right)$. The data confirm that neovascular targeting with EndoTAG-1 is a promising new method for the treatment of $\mathrm{PCa}$ by reducing the primary tumour mass and demonstrating benefits in the suppression of angiogenesis in comparison with the conventional treatment.
\end{abstract}

Correspondence to: Dr Lutz Trojan, Department of Urology, University Hospital Mannheim, D-68167 Mannheim, Germany

E-mail: lutz.trojan@uro.ma.uni-heidelberg.de

${ }^{*}$ Contributed equally

Key words: prostate cancer, chemotherapy, neovascular targeting, cationic liposomes

\section{Introduction}

Prostate cancer (PCa) is the most frequently diagnosed cancer in men in North America and Western Europe (1). The therapeutic concept of PCa conforms to the tumour stage, age and general condition of the patient. As a local $\mathrm{PCa}$ is curable by an operation or radiotherapy, the advanced or disseminated form of PCa is treated with androgen deprivation therapy in a palliative intention. However, most patients with an anti-hormonal therapy have a disease progression after a median of 13-20 month (2). After failure of an anti-hormonal therapy, the options for reducing the tumour mass are limited by a lack of effective therapy options.

Angiogenesis is necessary for tumour growth and metastasis (3). Tumour endothelial cells display higher proliferation rates than the endothelium in non-malignant tissue which make them more vulnerable for cytotoxic agents (4). 'Neovascular targeting' is an established approach in cancer therapy by destructing the tumours microvasculature (5). This concept was already demonstrated to be successful in preclinical studies for PCa with different antiangiogenic molecules $(6,7)$.

Paclitaxel is a microtubule-stabilizing antineoplastic cytotoxic drug which has antiangiogenic activity (8). It is approved for the treatment of different advanced solid cancers like metastatic ovarian and breast cancer and nonsmall cellular lung carcinoma. Currently, an application in PCa therapy exists only within study protocols. Because of the lipophilic character of paclitaxel, it is used with the solvent Cremophor EL, which provokes serious side-effects like neurotoxicity and hypersensitivity reactions.

Liposomes were first discovered as a reproducible and efficient method to introduce plasmid DNA into cells (9). Since then, liposomes represent an established drug delivery system for lipophilic substances by improving the therapeutic index and increasing the application dose of antitumoural agents (10). However, the clinical usage is impaired by the rapid clearance of liposomes by the reticuloendothelial system and their low bioavailability (11). In contrast to these conventional liposomes, positively charged cationic liposomes were originally developed for non-viral gene transfer into mammalian cells in vitro. On the background of 
negatively charged capillary endothelium in tumours (12), cationic liposome preparations were evaluated to be preferentially bound to and internalized by angiogenic negatively charged endothelial cells in tumours and chronic inflammation (13). Recently, cationic liposomes loaded with chemotherapeutic drugs have been evaluated as delivery vehicle for in vivo targeting of drugs to the tumour vasculature $(14,15)$.

In this study, we investigated the concept of neovascular targeting with paclitaxel encapsulated in cationic liposomes in an animal model as a new approach for advanced PCa. Therefore, we compared the macro- and microscopic aspects of tumour progression of the novel and a conventional therapeutic concept.

\section{Materials and methods}

Animal model. The trial was performed in accordance with the approved institutional protocol and the guidelines of the Institutional Animal Care and Use Committee. The Dunning rat PCa model is established for the evaluation of new treatment options against $\mathrm{PCa}$ (16). Male Copenhagen rats (Harlan-Winkelmann, Borken, Germany) were used in this study. After four weeks of acclimatisation, the animals were kept in separate cages after implantation of a central venous catheter.

Cell line. The Dunning R3327H rat prostatic adenocarcinoma subline MatLu was obtained from the ECACC (European Collection of Cell Cultures, catalogue number 94102735, Salisbury, UK). This subline displays a high metastatic ability with no androgen sensitivity and spreads almost exclusively via blood vessels into the lung. The tumour doubling time is estimated to be $2.7 \pm 0.3$ days in vitro and $3 \pm 0.2$ days in vivo (17).

The cells were cultivated at $37^{\circ} \mathrm{C}, 5 \% \mathrm{CO}_{2}$ and $100 \%$ humidity in $10 \mathrm{ml}$ RPMI-1640 medium (Invitrogen, Paisley, Scotland, UK) supplemented with $1 \mathrm{ml} 10 \%$ fetal bovine serum (PAN Biotech, Aidenbach, Germany), $0.2 \mathrm{ml} 2 \mathrm{mM}$ glutamine and $2.8 \mu 1250 \mathrm{nM}$ dexamethasone (both from Sigma, Taufkirchen, Germany).

Tumour implantation. Cell suspensions of $5 \times 10^{6}$ cells $/ \mathrm{ml}$ were generated and $1 \times 10^{6}$ cells were injected subcutaneous into the right flank region of the rat with a $25 \mathrm{G}$ cannula (Becton-Dickinson, Heidelberg, Germany) under general anaesthesia with isoflurane (Forene ${ }^{\circledR}$, Abott, Wiesbaden, Germany).

Drug preparation. EndoTAG-1 (Munich Biotech, Munich, Germany) and Paclitaxel (Bristol-Myers Squibb, Munich, Germany) were applied at a dose of $5.0 \mathrm{mg} / \mathrm{kg}$ b.w. Empty cationic liposomes (without paclitaxel) were provided by Munich Biotech and administered at the same lipid concentration as EndoTAG-1. All preparations were given volume adapted.

The treatment protocol contained an i.v. injection volume of $12 \mu \mathrm{l} / \mathrm{g}$ b.w. per animal administered over a $3 \mathrm{~min}$ period under general anaesthesia with isoflurane. EndoTAG-1 and empty liposomes, which were stored as lyophilisate at $4^{\circ} \mathrm{C}$, were prepared for injection by addition of $13.8 \mathrm{ml}$ aqua ad injectabilia. Paclitaxel was solved in 5\% glucose (B. Braun, Melsungen, Germany).

Treatment protocol. After tumour implantation on day 0 and tumour growth for 10 days, $24 \mathrm{~h}$ prior to the beginning of treatment, the animals received a central venous catheter (PAE-microtube $0.58 \times 0.96 \mathrm{~mm}$, NeoLab, Heidelberg, Germany) in the left V. femoralis under general anaesthesia with ketamine $\left(100 \mathrm{mg} / \mathrm{kg} / \mathrm{b} . w\right.$. i.p.; Hostaket ${ }^{\circledR}$, Intervet, Wiesbaden, Germany) and Xylazine ( $3 \mathrm{mg} / \mathrm{kg} / \mathrm{b} . w$. i.p.; Rompun $2 \%{ }^{\circledR}$, Bayer, Leverkusen, Germany). The animals were randomized into four groups $(\mathrm{n}=5$ in $\mathrm{Pax}, \mathrm{Cl}$ and $\mathrm{Gl}$ group; $n=6$ in EndoTAG-1 group) and were treated with either 5\% glucose (Gl), Pax, cationic liposomes (CL), or Pax encapsulated in cationic liposomes (EndoTAG-1) on day 12, 14,16 and 19.

Besides the evaluation of the general animal condition and nutritional status including body weight, the tumour was measured in three levels by calliper and volume which was calculated according to the formula $\mathrm{V}=\mathrm{a} \times \mathrm{b} \times \mathrm{c} \times 0.5$.

Immediately after euthanisation of the animals on day 20 , gross necropsy was performed with special emphasis on tumour, lungs, liver, kidneys and spleen. The tumour tissue was snap-frozen and stored at $-80^{\circ} \mathrm{C}$, the removed organs were fixed in $4 \%$ unbuffered formaldehyde (Merck, Darmstadt, Germany) and embedded in paraffin by a tissue embedding system (Leica TP $1020^{\circledR}$, Wetzlar, Germany).

Microvessel density. The cryo-perservated tumour tissue was sectioned at $6 \mu \mathrm{m}$ thickness and transferred to SuperFrostplus ${ }^{\circledR}$ slides (Langenbrinck, Emmendingen, Germany). After drying overnight, the slides were fixed for $10 \mathrm{~min}$ in acetone and subsequently air-dried.

The microvasculature of the tumour was evaluated by immunohistochemical staining of von Willebrand factor: To block endogenous peroxidase activity the sections were treated with $0.3 \%$ hydrogen peroxide in methanol for $30 \mathrm{~min}$ and rinsed in PBS $(\mathrm{pH}=7.0)$. The tumour tissue was incubated with avidine and after PBS washing with biotine (Avidine-Biotine-Blocking Kit ${ }^{\circledR}$, Linaris, Wertheim-Bettinger, Germany) for $15 \mathrm{~min}$ respectively. Followed by washing with PBS, the slides were incubated with goat serum (ABCkit Elite PK-6101 ${ }^{\circledR}$, Vector Laboratories, Burlingame, CA, USA) for 30 min. Polyclonal rabbit anti-human von Willebrand factor (PAK010M ${ }^{\circledR}$, Linaris) was used as the primary antibody in a dilution of 1:500 for $1 \mathrm{~h}$. Before incubating with the biotinylated goat anti-rabbit as the secondary antibody (ABC-kit Elite PK-6101) for $30 \mathrm{~min}$, the sections were rinsed in PBS. The tissue was coated with avidine-biotine-complex for $30 \mathrm{~min}$. The sections were finally stained with diaminobenzidine (DAB-Peroxidase kit SK- $4100^{\circledR}$, Vector; CA, USA) and washed in water. Last, the sections were counterstained with haematoxylin, dehydrated, and mounted.

The evaluation of microvessel density (MVD) was determined in the area of highest vascularisation. These vascular 'hot spots' were selected by scanning the tumour section at a low magnification $(x 40)$. Vessels were counted within the three highest vascularised areas by one investigator at a 


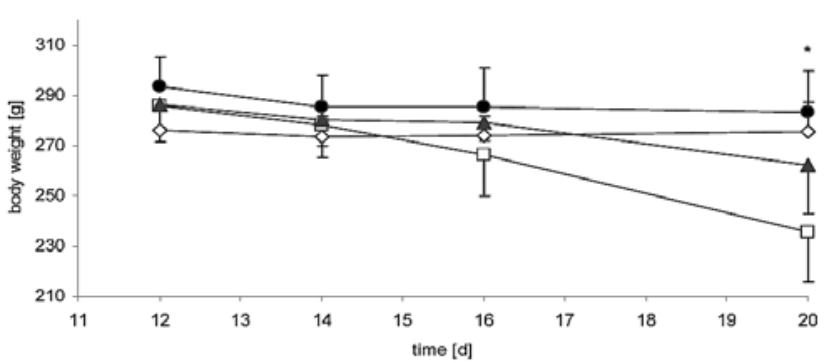

Figure 1. Development of mean body weight between the four groups G1 $(\diamond)$, EndoTAG-1 $(\square)$, Pax $(\bullet)$ and CL $(\bullet)$ during treatment. No difference was observed between the four groups on day 12, 14 and 16. On day 20 animals in EndoTAG-1 and Pax group revealed a significant decrease in body weight $\left({ }^{*} \mathrm{p}<0.002\right.$ EndoTAG-1 and Pax vs. CL and Gl; variance analysis).

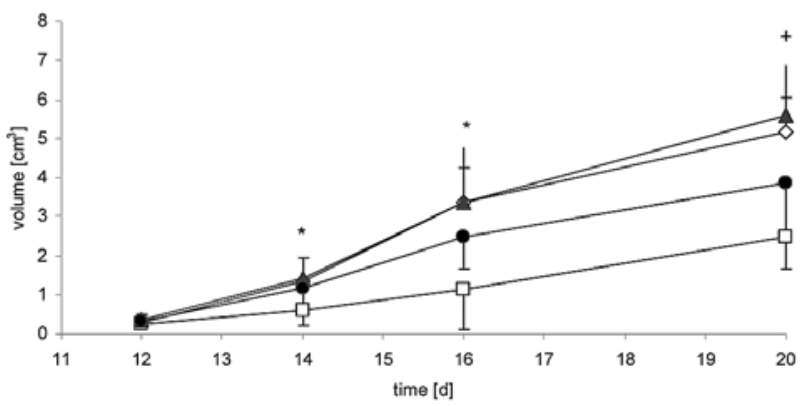

Figure 2. Development of mean tumour volume between the four groups Gl $(\diamond)$, EndoTAG-1 $(\square)$, Pax $(\bullet)$ and CL $(\bullet)$ during treatment. Significant difference in tumour volume between the groups were observed from the second day of therapy (day $14 \mathrm{p}<0.138$; day $16 \mathrm{p}<0.006$; day $20 \mathrm{p}<0.003$; variance analysis). EndoTAG-1 group developed the significant smallest tumours vs. all other treatment arms on day $14\left({ }^{*} \mathrm{p}<0.018\right)$ and day 16 $\left({ }^{*} \mathrm{p}<0.002\right)$; EndoTAG-1 vs. Gl and Pax on day $20\left({ }^{+} \mathrm{p}<0.003\right.$; all F-test $)$.

magnification of x200. The average microvessel density was measured in vessels per $\mathrm{mm}^{2}$ by a standardised counting grid. This protocol constitutes an established method for the quantification of MVD (18).

Histopathology. Organ sections of $3 \mu \mathrm{m}$ were deparaffinated and rehydrated according to standard procedures and soaked in haematoxylin and water for $10 \mathrm{~min}$. The sections were stained in $0.1 \%$ eosin for $2 \mathrm{~min}$, dehydrated and mounted.

Statistics. The statistical analysis was performed using SAS (Release 8.02; SAS Institute Inc., Cary, NC, USA). Results are presented as mean $\pm \mathrm{SEM}$. $\mathrm{P}<5 \%$ was considered to be significant.

An ANOVA was used to show differences between the four treatment groups for the body weight of animals and for the tumour volume. Scheffe's test compared the body weight of the EndoTAG-1- and Pax group. Dunnett's test, F-test and t-test investigated differences in tumour volume between EndoTAG-1 group and the other groups. Kruskal-Wallis-Test and Mann-Whitney U-test were used to compare the MVD between the therapy groups.

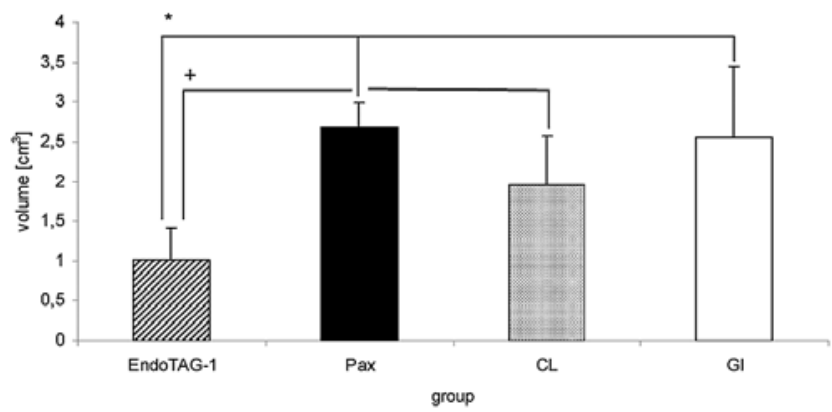

Figure 3. Group-specific mean tumour volume over the whole treatment period. EndoTAG-1 treated tumours possessed the smallest volume ( ${ }^{*} \mathrm{Gl}$ vs. EndoTAG-1 p=0.01; *Pax vs. EndoTAG-1 p=0.01; ${ }^{+}$Liposome vs. EndoTAG-1 p=0.05; Dunnett's test).

\section{Results}

Every animal developed a palpable subcutaneous tumour in the right flank region between day 7 and 10 after cell implantation. All animals finished the trial except for one animal in the EndoTAG-1 group. Due to the distinct deterioration of general condition and nutritional status, it was euthanized on day 17 .

At the beginning of the i.v. treatment protocol on day 12, there were no differences in general condition and body weight between the four groups $(285 \pm 12.8 \mathrm{~g}$; $\mathrm{p}=0.20$, variance analysis).

Animals which were treated with glucose $(274.4 \pm 11.8 \mathrm{~g})$ and pure cationic liposomes $(283.2 \pm 16.1 \mathrm{~g})$ finished treatment without a significant change of the body weight. Body weight of animals in the chemotherapeutic groups was reduced significantly during treatment (day 20: EndoTAG-1 235.6 $\pm 19.9 \mathrm{~g}, \mathrm{p}=0.0002 ;$ Pax 262.2 $\pm 19.5 \mathrm{~g}, \mathrm{p}=0.0237$, variance analysis) without a significant difference between the EndoTAG-1 and the Pax group ( $\mathrm{p}>0.05$, Scheffe's test) (Fig. 1).

Histopathology. On histopathological examination of organs, the majority of animals showed, regardless of group affiliation, mural thrombus consisting of fibrin, inflammatory cells, detritus and tumour cells.

The status of the lymphatic system in animals of the EndoTAG-1 and Pax group differed from the control groups. It displayed an absence of lymphocytes in lymph nodes and lymphatic tissues whereas the animals from GL and CL groups showed signs of an activated immune response.

Tumour progression. At the beginning of the treatment (day 12), there was no interrelation between body weight and tumour volume ( $\mathrm{p}=0.38, \mathrm{r}=0.21$, variance analysis) and no difference in tumour volume between all groups $(0.31 \pm$ $0.12 \mathrm{~cm}^{3}, \mathrm{p}=0.68$ variance analysis). Significant differences of the tumour volume between the groups were observed from the second day of therapy (day 14 p $<0.138$; day 16 $\mathrm{p}<0.006$; day $20 \mathrm{p}<0.003$; all variance analysis). At the end of the trial (day 20), lowest tumour volume was found in the EndoTAG-1 group $\left(2.49 \pm 0.8 \mathrm{~cm}^{3}\right)$ followed by CL $(3.87 \pm$ $\left.1.3 \mathrm{~cm}^{3}\right)$, Gl $\left(5.17 \pm 2.1 \mathrm{~cm}^{3}\right)$ and $\operatorname{Pax}\left(5.59 \pm 0.5 \mathrm{~cm}^{3}\right)$. EndoTAG-1 treated tumours possessed the significantly 


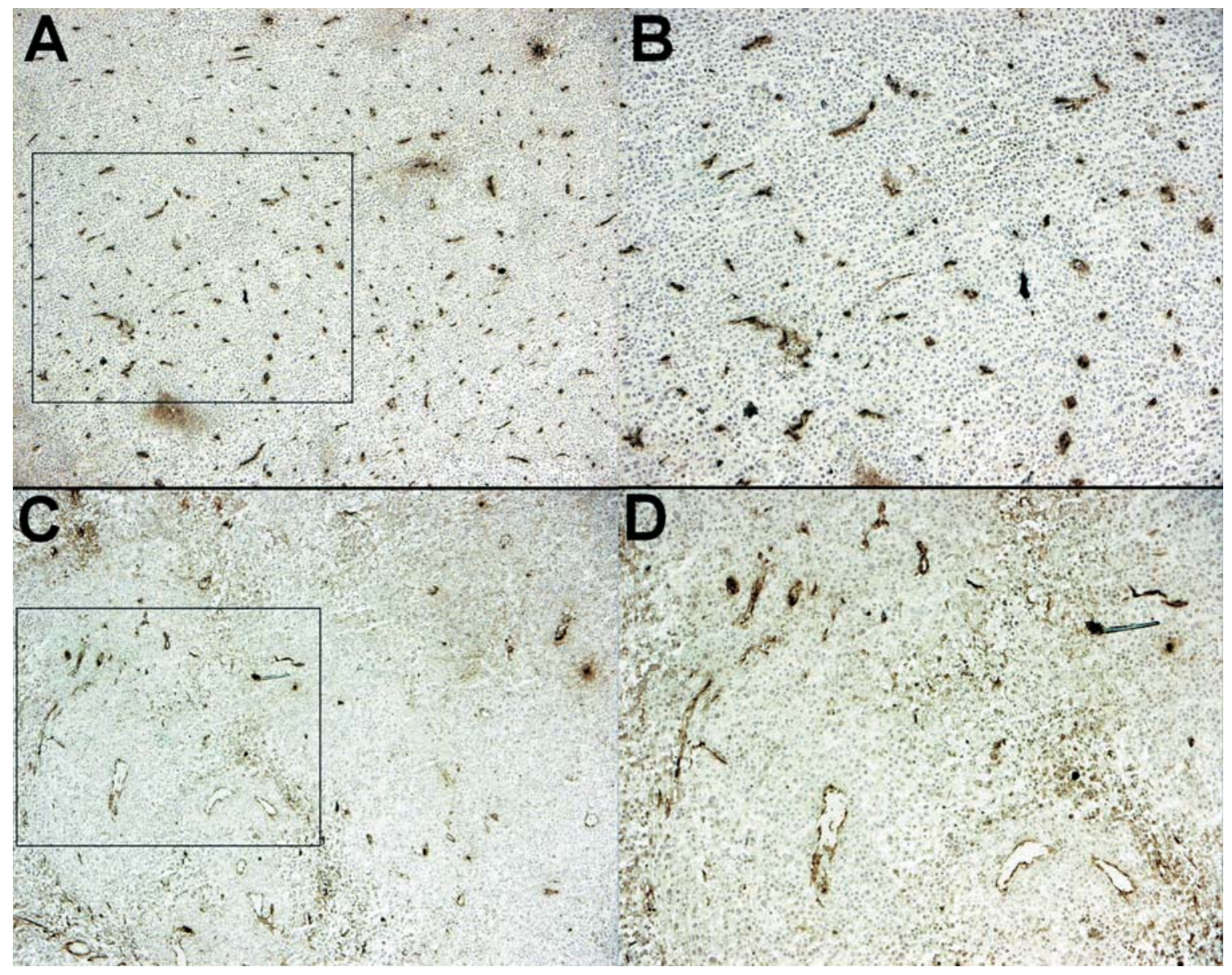

Figure 4. Immunohistology of tumour tissue. Endothelial cells were stained with vWF antibodies to evaluate MVD of the tumour. The vascular 'hot spots' were shown in low magnification (x40) on the left (A and C) and in high magnification (x200) on the right (B and D). Highest MVD was found in the CL group (A and B), EndoTAG-1 treated tumours revealed the lowest MVD (C and D).

smallest tumour volumes compared with Gl (day $14 \mathrm{p}<0.008$; day $16 \mathrm{p}<0.001$; day $20 \mathrm{p}<0.002$ ) and Pax (day $14 \mathrm{p}<0.004$; day $16 \mathrm{p}<0.001$; day $20 \mathrm{p}<0.001)$. In contrast to $\mathrm{CL}$ group, the neoplasms in the EndoTAG-1 group were significantly smaller on day $14(\mathrm{p}<0.033)$ and day $16(\mathrm{p}<0.011)$, but not on day 20 ( $<<0.077$; all t-test) (Fig. 2). The group-specific mean tumour volume over the whole treatment differed significantly (EndoTAG-1 $1.01 \pm 0.4 \mathrm{~cm}^{3}, \mathrm{Cl} 1.96 \pm 0.6 \mathrm{~cm}^{3}, \mathrm{Gl}$ $2.55 \pm 0.8 \mathrm{~cm}^{3}$, Pax $2.69 \pm 0.3 \mathrm{~cm}^{3}, \mathrm{p}=0.0008$, variance analysis). The mean tumour volume in the EndoTAG-1 group was significantly smaller than in all other groups $(\mathrm{Gl} p=0.01$; Pax $\mathrm{p}=0.01$; CL=0.05; Dunnett's test) (Fig. 3).

The MVD was heterogeneous within the groups, so the results showed only a trend between the four treatment arms $(\mathrm{p}=0.0521$, Kruskal-Wallis-Test). Lowest MVD was found in the EndoTAG-1 group $\left(11.78 \pm 2.68\right.$ vessels $\left./ \mathrm{mm}^{2}\right)$ followed by Gl $\left(15.64 \pm 6.68\right.$ vessels $\left./ \mathrm{mm}^{2}\right)$, Pax $(18.22 \pm 9.50$ vessels/ $\left.\mathrm{mm}^{2}\right)$ and CL $\left(40.9 \pm 32.8\right.$ vessels $\left./ \mathrm{mm}^{2}\right)$, thereby EndoTAG-1 and CL significantly differed in their MVD to the other two groups ( $\mathrm{p}=0.032$, Mann-Whitney U-test) (Fig. 4).

The formation of metastases in the tumour model was confirmed in this study. With exception of one case of a solid metastasis in perirenal fatty tissue (Gl group), metastatic cancer cells were exclusively found within the lung of the animals. All animals developed lung metastases except for one animal in the Pax group. The distribution of solid metastases and diffuse tumour cells was homogeneous within and between the four treatment arms.

\section{Discussion}

Therapeutic options for advanced prostate cancer after androgen deprivation therapy are still limited. Taxane based chemotherapy has demonstrated to extend survival especially in combination with novel agents like antiangiogenic drugs (19).

A recent strategy in the treatment of neoplasms is to attack the tumour microvasculature as suggested by Denekamp et al (5). The approach of specific inhibition of the tumour supplying vessels is supported by the genetically stability of endothelial cells in comparison to neoplastic cells. Without blood vessels, tumours are not able to grow beyond a critical size or metastasize to other organs. Furthermore, the destruction of $>100$ tumour cells by deletion of one endothelial cell is an efficient way of targeted anticancer therapy (20).

The inhibition of endothelial cell proliferation can be managed with the taxane paclitaxel, a chemotherapeutic drug with an antiangiogenic activity (8). The treatment with paclitaxel has already been evaluated to prolong mean 
survival time in advanced PCa $(21,22)$. However, the therapy is limited by its low therapeutic index and the serious sideeffects of the drug itself and the conventional solvent Cremophor EL.

Cationic liposomes were first utilised in gene therapy for application of DNA in vivo, later their accumulation in activated endothelial cells was observed (23). Thurston et al have shown that angiogenic endothelial cells exhibit a preferential uptake of cationic liposomes by endocytosis (13). The negatively charged glycocalyx of proliferating endothelial cells is suggested to promote the selective uptake of cationic particles. Further investigations have revealed a selective accumulation and prolonged retention in tumour tissue and vasculature for positive charged liposomes whereas neutral and anionic liposomes did not show these attributes (24).

In this study, we evaluated the effect of paclitaxel encapsulated in cationic liposomes in hormone refractory and disseminated $\mathrm{PCa}$ in comparison to the commercial available formulation form of paclitaxel. During treatment, the animals in the chemotherapeutic groups (Pax and EndoTAG-1) suffered from reduced general condition which was reflected in a loss of body weight. Because of the normal nutritional condition in the control groups and the predescribed mean survival time of $35 \pm 1$ days after tumour implantation (17), the partly serious reduction of the general condition towards the end of treatment is supposed to be substantiated by chemotherapy without an emphasis on the form of drug delivery. Toxicity studies with intravenous application of comparable dosages of paclitaxel on rats revealed a loss of body weight and a reduced food intake (25). Furthermore, the described loss of infection defence, myelosuppression and hypoplasia of lymphatic organs agrees with the histopathological findings in this study.

Our experiment showed the strongest inhibition of tumour growth in the EndoTAG-1 treated animals compared with the standard paclitaxel treatment and the control groups. Surprisingly, together with Gl group, Pax treated animals displayed the highest tumour volume of all groups despite the fact that paclitaxel was applied in equal amounts in both chemotherapeutic arms. A previous study that investigated the efficacy of EndoTAG-1 and soluble paclitaxel under a similar treatment protocol in a Syrian Golden hamster melanoma model revealed controversial results (26). In our study, we did not evaluate the effect of the formulations of paclitaxel in MatLu-cells in vitro, however, an equal antiproliferative effect was proven for a melanoma mouse model in vitro (27).

The effect of EndoTAG-1 on metastases was missing in contrast to the already described deceleration of incidence of metastases. Filiae of MatLu-tumours are valid to response to chemotherapy, but only a quantitative reduction was described (28). So, the cause for the lack of differential results between the therapy arms could exist in the exclusive qualitative evaluation of the metastatic status.

The previously observed antitumoural efficacy of empty cationic liposomes was confirmed by our findings (29). On day 20, the tumour volume in the CL group was even smaller than in the Pax group. But the significant differences in MVD for EndoTAG-1 and CL groups do not prove an antiangiogenic mechanism of action responsible for the therapeutic effect of empty cationic liposomes. In our study due to the observed strong variation of MVD within the control groups a statistically significant antivascular action of EndoTAG-1 superior to the paclitaxel or glucose control could not be demonstrated. Nevertheless EndoTAG-1 showed the strongest antiangiogenic activity which reached significance against the $\mathrm{Cl}$ group. Previous studies with a comparable experimental setup in other tumour entities were able to show a statistically significant EndoTAG-1 related inhibition of angiogenesis compared to other treatment arms $(26,27)$.

In conclusion, our data suggest that paclitaxel encapsulated in cationic liposomes is able to reduce tumour growth significantly in comparison to the standard application formula. The reduction of side-effects and a suppression of metastasis by the treatment with EndoTAG-1 were not confirmed in this study. The microvessel density was the least in EndoTAG-1 group with a weak significance compared to conventional Paclitaxel suggesting an antiangiogenic action of EndoTAG-1 in this rat tumour model.

Our study demonstrates that cationic liposomes can increase the therapeutic index of paclitaxel by reducing the primary tumour mass in an animal model. The therapeutic effect may be accomplished by reduced angiogenesis.

\section{References}

1. Farkas A, Schneider D, Perrotti M, et al: National trends in the epidemiology of prostate cancer, 1973 to 1994: evidence for the effectiveness of prostate-specific antigen screening. Urology 52: 444-449, 1998.

2. Sharifi N, Dahut WL, Steinberg SM, et al: A retrospective study of the time to clinical endpoints for advanced prostate cancer. BJU Int 96: 985-989, 2005.

3. Folkman J: The role of angiogenesis in tumor growth. Semin Cancer Biol 3: 65-71, 1992.

4. Carmeliet P and Jain RK: Angiogenesis in cancer and other diseases. Nature 407: 249-257, 2000.

5. Denekamp J: Endothelial cell proliferation as a novel approach to targeting tumour therapy. Br J Cancer 45: 136-139, 1982.

6. Borgstrom P, Bourdon MA, Hillan KJ, et al: Neutralizing antivascular endothelial growth factor antibody completely inhibits angiogenesis and growth of human prostate carcinoma micro tumors in vivo. Prostate 35: 1-10, 1998.

7. Miki T, Nonomura N, Nozawa M, et al: Angiogenesis inhibitor TNP-470 inhibits growth and metastasis of a hormoneindependent rat prostatic carcinoma cell line. J Urol 160: 210-213, 1998.

8. Belotti D, Vergani V, Drudis T, et al: The microtubule-affecting drug paclitaxel has antiangiogenic activity. Clin Cancer Res 2: 1843-1849, 1996.

9. Felgner PL, Gadek TR, Holm M, et al: Lipofection: a highly efficient, lipid-mediated DNA-transfection procedure. Proc Natl Acad Sci USA 84: 7413-7417, 1987.

10. Perez-Soler R: Liposomes as carriers of antitumor agents: toward a clinical reality. Cancer Treat Rev 16: 67-82, 1989.

11. Poste G, Bucana C, Raz A, et al: Analysis of the fate of systemically administered liposomes and implications for their use in drug delivery. Cancer Res 42: 1412-1422, 1982.

12. Ran S, Downes A and Thorpe PE: Increased exposure of anionic phospholipids on the surface of tumor blood vessels. Cancer Res 62: 6132-6140, 2002.

13. Thurston G, McLean JW, Rizen M, et al: Cationic liposomes target angiogenic endothelial cells in tumors and chronic inflammation in mice. J Clin Invest 101: 1401-1413, 1998.

14. Schmitt-Sody M, Strieth S, Krasnici S, et al: Neovascular targeting therapy: paclitaxel encapsulated in cationic liposomes improves antitumoral efficacy. Clin Cancer Res 9: 2335-2341, 2003. 
15. Eichhorn ME, Luedemann S, Strieth S, et al: Cationic lipid complexed camptothecin (EndoTAG( (R))-2) improves antitumoral efficacy by tumor vascular targeting. Cancer Biol Ther 6: 920-929, 2007.

16. Smolev JK, Heston WD, Scott WW, et al: Characterization of the Dunning $\mathrm{R} 3327 \mathrm{H}$ prostatic adenocarcinoma: an appropriate animal model for prostatic cancer. Cancer Treat Rep 61: 273-287, 1977.

17. Isaacs JT, Isaacs WB, Feitz WF, et al: Establishment and characterization of seven Dunning rat prostatic cancer cell lines and their use in developing methods for predicting metastatic abilities of prostatic cancers. Prostate 9: 261-281, 1986.

18. Hasan J, Byers R and Jayson GC: Intra-tumoural microvessel density in human solid tumours. Br J Cancer 86: 1566-1577, 2002.

19. Sonpavde G and Hutson TE: New approaches in hormone refractory prostate cancer. Am J Clin Oncol 29: 196-201, 2006.

20. Folkman J: Angiogenesis in cancer, vascular, rheumatoid and other disease. Nat Med 1: 27-31, 1995.

21. Petrylak DP, Macarthur RB, O'Connor J, et al: Phase I trial of docetaxel with estramustine in androgen-independent prostate cancer. J Clin Oncol 17: 958-967, 1999.

22. Kelly WK, Curley T, Slovin S, et al: Paclitaxel, estramustine phosphate, and carboplatin in patients with advanced prostate cancer. J Clin Oncol 19: 44-53, 2001.

23. Zhu N, Liggitt D, Liu Y, et al: Systemic gene expression after intravenous DNA delivery into adult mice. Science 261: 209-211, 1993.
24. Krasnici S, Werner A, Eichhorn ME, et al: Effect of the surface charge of liposomes on their uptake by angiogenic tumor vessels. Int J Cancer 105: 561-567, 2003.

25. Kadota T, Chikazawa H, Kondoh $\mathrm{H}$, et al: [Toxicity studies of paclitaxel. (II)-One-month intermittent intravenous toxicity in rats]. J Toxicol Sci 19 (Suppl 1): 11-34, 1994.

26. Strieth S, Eichhorn ME, Sauer B, et al: Neovascular targeting chemotherapy: encapsulation of paclitaxel in cationic liposomes impairs functional tumor microvasculature. Int J Cancer 110: 117-124, 2004.

27. Kunstfeld R, Wickenhauser G, Michaelis U, et al: Paclitaxel encapsulated in cationic liposomes diminishes tumor angiogenesis and melanoma growth in a 'humanized' SCID mouse model. J Invest Dermatol 120: 476-482, 2003.

28. Lazan DW, Heston WD, Kadmon D, et al: Inhibition of the R3327MAT-Lu prostatic tumor by diethylstilbestrol and 1,2-bis (3,5-dioxopiperazin-1-yl)propane. Cancer Res 42: 1390-1394, 1982.

29. Dass CR: Immunostimulatory activity of cationic-lipid-nucleicacid complexes against cancer. J Cancer Res Clin Oncol 128: 177-181, 2002. 